\title{
Design and testing booking application for private doctors' practices to support disaster management
}

\author{
Dalila Husna Yunardi', Rahmad Dawood ${ }^{2}$, Maya Fitria $^{1}$, Rini Deviani ${ }^{2}$ \\ ${ }^{1}$ Department of Engineering, Syiah Kuala University, 23111 Darussalam, Banda Aceh, Indonesia \\ ${ }^{2}$ Department of Informatics, Syiah Kuala University, 23111 Darussalam, Banda Aceh, Indonesia
}

\begin{abstract}
During the pandemic or natural disaster, people will be dispersed and locked down in their own homes or crisis centres. During these times, people are still in need of access to healthcare. While general healthcare would be provided by the government during these times, certain people within the population will need access to specialised doctors, especially children, the elderly and people who have prior or longterm diseases. During a disaster, people may not have access to these services. Therefore, a simple technology that lets them book an appointment with these specialised doctors will be designed in this research. This booking application, will be able to back up the general healthcare when it is overwhelmed, especially during a disaster. This research was carried out using qualitative research. The data collection was conducted using interview and observation. These primary data are then coded according to the general themes. The data is then used to create a usable design for the booking application prototype, using UserCentred Design as its method. After the prototype was created successfully, it was then tested for its usability with its potential users. 6 people from 3 user groups agreed to be respondents for the usability testing. Using think aloud protocols, it was found that on average 4 out of 6 people found the prototype usable and suitable for their jobs.
\end{abstract}

\section{Introduction}

Indonesia has different healthcare system compared to other countries in the world. The current healthcare system, dictates that patient must first go to a community health centre to be diagnosed. Then get a recommendation to see specialised doctor according to the first diagnosis made by a general practitioner in the health community centre. Patients can use the national healthcare insurance known as BPJS, that will provide them with free services.

However, sometimes patients feel as if they do not get the best service provided with BPJS, or they want more time with the specialised doctors; therefore, they have the option to go to practices in private clinics in order to see the doctors that they want. This can be achieved by calling the phone number of the desired practice and register to see the doctor. The patients are usually are given a number and they must wait before the doctor can attend to them.

This way of seeing the doctor is quite common in Banda Aceh. Some people do not want to go to the health community centres first, due to the longer waiting time and the lack of specialised doctors. In most cases, older patients are seen to prefer to go to private practices as they would like to see the same doctors for their ailments.
However, during disaster time or times like this; pandemic, it would be a lot harder to go to the private practices and wait in line. Most people are afraid to go to the private practices, as some doctors still share the space with other doctors and there are also other people waiting in line. This will increase the likelihood of being in contact with diseases that normally spread with disasters or to COVID-19. This can be managed using technology.

Thus, the research aims to design and test a booking application that caters for registering patients who would like to make an appointment with their doctors. This application will support private clinics in managing their time as well as the crowds, as people still get ill during disaster and pandemic, which means they still need to go and see their doctors.

\subsection{Research objectives}

The objectives of this research are as follows:

- To collect relevant data from prospective users of specialised doctor's private practices in Banda Aceh

- To observe the activities that are carried out in a day within the private practice

- To interview and confirm the activities that took place in the private practice

*Corresponding author: dalilayunardi@unsyiah.ac.id 
- To process and code the data

- To apply user-centred design in building a prototype design for booking application, and

- To conduct usability testing for the prototype to establish user satisfaction.

\section{Research methodology}

The development of this application will focus on user experiences, which means having a deep understanding of related users, what they need, what they value, their abilities, and also their limitations on utilizing the application. Hence, this research will be conducted using qualitative methodology for the data collection and analysis. This methodology will be used alongside User-Centred Design; a methodology to design software.

\subsection{Qualitative research}

Shank [1] defined qualitative research as a method in which experiences of the subjects are taken systematically where those experiences will then create meaning. Shank [1] also emphasised that by choosing qualitative research methodology, one must abide by the rules set and agreed upon the people within the qualitative research community. Quantitative research focuses on meanings and the context of a phenomenon [2], which in turn supports the methodology that will be used to design the software; namely User-Centred Design (UCD). UCD requires its developer to understand what the user needs' are in order to design and build a usable software [3], therefore this type of research fits the profile of the identity of this research: interpretative and naturalistic [4].

Qualitative research provides an in depth understanding as well as interpreting meanings from phenomenon within their natural settings. These factors will contribute heavily in this research, since this research looks well into how the current situation of specialised doctor's practise are run in a day-to-day basis [4].

The sections below will explain the techniques that will be used in this research, they are: interview and observation. The later sections will also explain the software development methodology as well as the usability testing techniques.

\subsubsection{Observation}

Observation is one of the oldest and most fundamental of qualitative research techniques. Observation is commonly accompanied by either interview, which is also conducted in this research and document analysis [5].

Observation is when the researcher collects data by looking and listening as well as using other senses to make meaning out of a phenomenon. The process is carried out systematically and purposefully [5].

During observation, the researcher takes particular note on the activities that are carried out in a phenomenon, the actors or people involved, the sociodemographic of the people, the setting or the environment in which the phenomenon takes place and the relationship between people within the phenomenon. These things are some of the items that the researcher will take note during the course of the observation [6].

The researcher records any interactions, process or knowledge that took place whilst observation. These items are known as fieldnotes; which will act as primary data for the researcher to work on [5].

Observation ties in with UCD, as data from observation provides how the world is perceived in its actual settings. Observation will give sense towards the application that is about to be designed.

The observation took place in 3 specialised doctor's practices in the city of Banda Aceh while adhering to strict health protocols suggested by the local authorities.

\subsubsection{Interview}

Interview is a conversation that takes place between the interviewer and the interviewee. During this conversation, knowledge is interchangeable between the two or more people who are interacting in the interview. In qualitative research, interview has become commonly used to make sense and meaning about a phenomenon. Researchers use interview to gain understanding about a process as well as to confirm any previous knowledge gathered using other techniques. Interview can be conducted in various ways, such as interviewing people by phone, written or online surveys, meeting people face to face and record the interview or direct in-person interviews, where the subjects are interviewed directly during an observation or when an activity takes place [7].

The recorded interviews are then transcribed and coded. Coding in qualitative research is the process of generating ideas and themes that occur during the interview. During coding, the researcher takes the unprocessed data and identify the underlying concepts and categories [8]. In this research, these categories and concepts are arranged in a pyramid-like structure to find the repeated events or themes.

The data obtained from the interview will confirm the findings from the observation session. The interview will provide meaning to the activities that the people are doing. This will in turn feed the data to the UCD methodology as design requirements.

The interview will be conducted with 6 people from 3 user groups. The user groups are, receptionists, patients and healthcare providers.

Due to the reduced activities for citizens of Indonesia during the COVID-19 pandemic, the interview for this research was conducted via video conference and it was recorded.

\subsection{User-Centred Design (UCD)}

User-centred design is a software design methodology where the interface of the system or application is created and planned according to the needs and capabilities of the people who are going to use it [9]. Figure 1 shows the activities that are conducted in UCD. 


\subsubsection{Think aloud protocol}

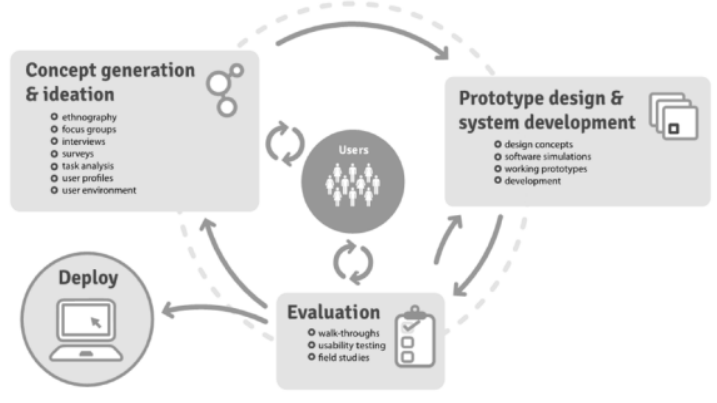

Fig. 1. User-centred design process, extracted from [10]

Figure 1 shows the various activities and stages that are conducted in UCD. The first stage is concept generation and ideation. This is where the researcher would collect qualitative data as necessary. In this research, this is done by conducting observation and interviews. The data collected from those two techniques then provide the vision of what the application would look like. Then based on the vision, the next stage of this method is to build a prototype design and system environment. In this stage, initial design concepts are introduced and a working prototype is built. Then the prototype is evaluated using usability testing with the prospective users. This stage is where the users are operating the prototype based on scenarios given. The process continues in a loop until the desired product is reached, then finally the end-product is released or deployed to the public [10].

The objective of UCD is that the design of an application is user data-driven, meaning the person in charge of the direction of the application should understand fully how the data interacted with the used before proposing a design [11].

By doing so, the expected outcome is that a design that will provide a rich and in context user experience for the users.

\subsection{Usability testing}

Usability testing is method that assesses the experience of users while operating a digital application [3]. In usability testing the user is given a set of tasks that $\mathrm{s} / \mathrm{he}$ must complete. This method is designed to look for any part of the application that give the most problems for the users, hence giving them an inadequate user experience.

While the researcher notes the findings, the design and the experience of the application can be re-evaluated and built to suit the user better.

In this research the usability testing will be conducted with think aloud protocol via online video conference application due to the COVID19 pandemic. The possible users of this application have agreed to be recorded and have given consent for their profiles to be analysed, as long as their identities remain anonymous.
Think aloud protocol is a technique within human computer interaction where the users articulate what they are thinking while carrying out tasks. Think aloud protocols are often used in training user experience (UX) professionals in their line of work and are considered as the golden standard of usability testing [12].

In this research think aloud protocol will have its own scenario devised by the researcher and will be conducted via online video conference application.

\subsection{Research Environment and Sampling}

The research is conducted during the COVID19 pandemic. There were 3 private practices being observed directly. These private practices are within the city of Banda Aceh.

Purposeful sampling is used to collect the samples in this research. Purposeful sampling is a way of selecting samples in qualitative research where the sample size is strategically determined to fit the needs of the research.

For the interview, there were 6 interviews conducted, where 2 of them were face to face; adhering to strict health protocols. The rest were done using online video conference software. All respondents agreed for their participation to be recorded. The 6 respondents include 2 patients, 2 security officers and 2 receptionists.

\section{Results and discussion}

The results of this research will be explained in further sections.

\subsection{Observation results}

There were 3 specialised doctor's practices that were observed in this research. They are: Cempaka Lima Clinic, NCB Clinic and Bunda Thamrin Clinic. These practices agreed to be observed in the afternoon where it is normally the peak time for patients to come for their appointments. These practices have specialised doctors from many fields, such as dermatology, oncology, obstetrics and gynaecology, dentistry, internist, ophthalmology and also a pharmacist is in place. These practices were located around Banda Aceh.

These 3 practices showed similarities in the registration process, waiting period and regulations during COVID-19 pandemic.

All 3 practices have receptionist desks up front after the front door. This is where the patients register, asked for information and check the doctor's schedule. 1 practice has a landline and the rest use a mobile phone to contact doctors and patients. 2 out of 3 practices have security officers as their first point of information and other things that are deemed important.

During observation, it was found that almost all patients have come earlier during the day to the practices to register themselves and book for an appointment for their preferred doctors. The receptionists take their 
personal details and write them in a book. Copies of the appointment list are distributed through the personal assistants or nurses in attending od each doctor. They will refer to this list when calling out patients' names.

Patients generally arrived about half an hour to an hour before the doctor arrives. Patients wait for their turn in the waiting room for their names. Despite the COVID-19 regulations, some patients still sit inside the waiting room. This is because they want to be there when the doctor arrives and have a greater chance of being called first even though they may be on the last of the list.

However, one of the practices' receptionists made remarks to the patients that they can wait outside or in their vehicles for the appointment times. When the doctor has arrived, they will be called through their mobile phones to attend to their appointments.

In all 3 private practices, they have printed out rules and regulations regarding the social distancing and health protocols that must be applied by all inbound patients coming into the practice. These rules are also stated by the receptionist during the registration process.

To conclude, the observation on the field revealed that the current registration process and waiting period are very much similar in every setting. Therefore, it is necessary to support this process through a technology.

\subsection{Interview results}

During the interview with the respondents were asked to describe these factors:

1. Short biography about themselves; this is to provide a demographic for the research.

2. Reasons for going to a specialised doctors' practices (patients).

3. The current registration procedure during COVID-19 pandemic (patients).

4. The situation during registration procedure (patients).

5. The working hour (receptionist and security officers).

6. Activities during working hour (receptionists and security officers).

7. Any other problems that they encounter during the registration and booking process.

Figure 2 shows the themes that were found during the interview.
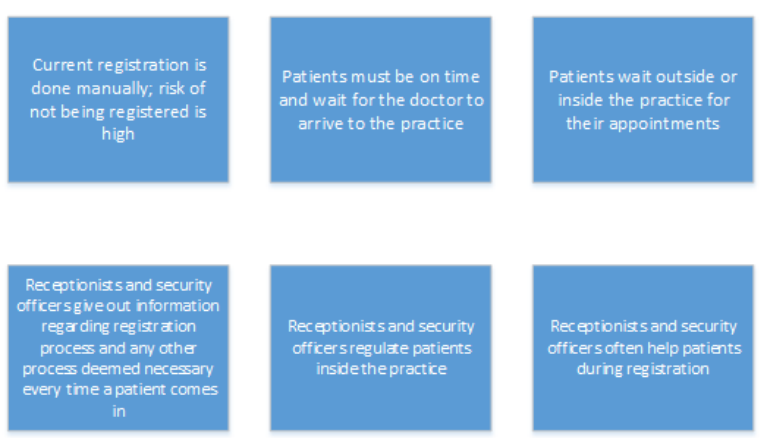

Fig. 2. themes discovered from interview process.
These themes represented the key areas that are considered to be problems within the private practices. One of the recurring themes is that the long waiting time after registration and seeing the doctor for the appointment. According to one of the patients interviewed (Male, 25), the earlier a patient registers for an appointment during that day, the quicker he gets to see the doctor.

This corroborates the waiting time between after registration time and meeting the doctor, which is about 10 minutes if the patient is an early bird. However, if the patient registers late, it is about 50 minutes up to an two hours before he can see the doctor. This also depends on the time spent between the doctor and the patients.

Figure 2 also suggests that the receptionists of the private practices had to regulate the amount of people coming into the practice. This also showed up during observation. People were waiting around the practice were waiting inside the practice for the doctor. One of the receptionists interviewed (Female, 22) mentioned that the patients stay around in the practice with the hope that they will be able to take someone else's time if another patient is late to his appointment.

Both of the security officers interviewed mentioned that they had to put extra work in regulating the patients who are coming into the practice. Due to COVID19 pandemic, there was a rule set up by the local council that people should be distanced about 2 metres from each other within public spaces. Therefore, there should a certain number of people within the private practices. However due to other illnesses that patients have and must be treated, sometimes this regulation is turned a blind eye upon.

All of the interviewees mentioned that it would be helpful if they had some sort of simple technology that can help them in their daily activities of registering and helping patients in the private practices.

\subsection{Prototype}

Based on the observation and interview results, a simple application can be developed. The results and themes from Figure 2 suggested that the most suitable technology for this research is a mobile application. The next section explains the visioning of the future booking application.

\subsubsection{Visioning}

Visioning in this case is when the functionalities of this application is proposed. According to tree diagrams in Figures 2, 3 and 4, the booking application should have 3 sides. The first side is the patient's side, then the receptionist's side and finally the doctor's or nurse's side. These 3 sides will support a more cohesive registration system that will regulate the amount of people coming in to see the specialised doctors.

This will also be a plus point during a disaster and a pandemic such as today. During a disaster, people may be displaced and confused. People with pre-existing disease may have difficulties in reaching their usual doctors. With the support of this proposed application, 
patients can reach and make appointments during a disaster or pandemic to see their doctors.

Figure 3 shows the proposed functionalities for the patient's side of the application. These functionalities are derived from the results of interview and observation.
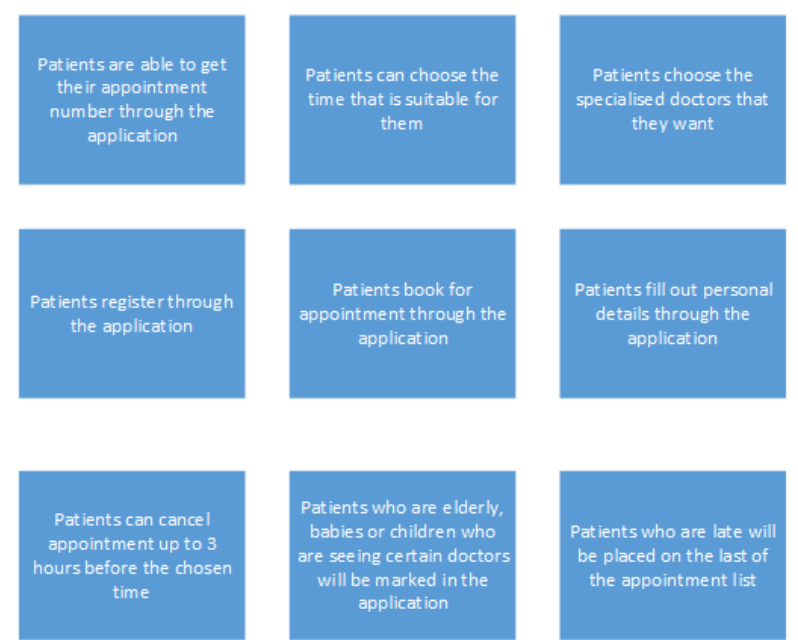

Fig. 3. Proposed functionalities for patient's side

Figure 4 shows the proposed functionalities for the receptionist's side of the application. These functionalities are derived from the results of interview and observation.
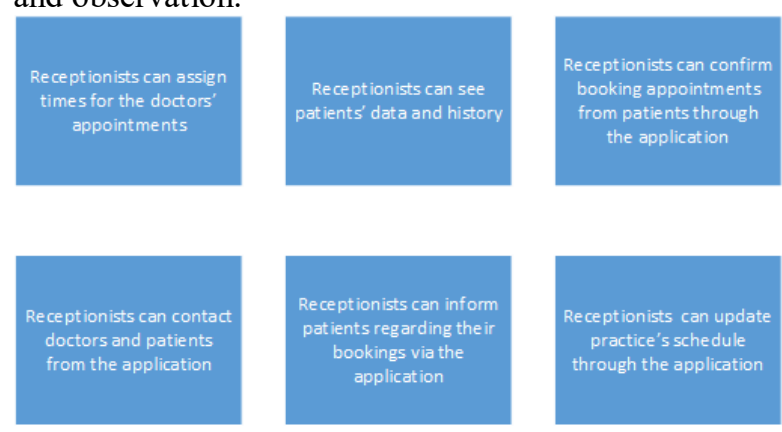

Fig. 4. Proposed functionalities for doctor's side

Figure 5 shows the proposed functionalities for the doctor's or nurse's side of the application. These functionalities are derived from the results of interview and observation.
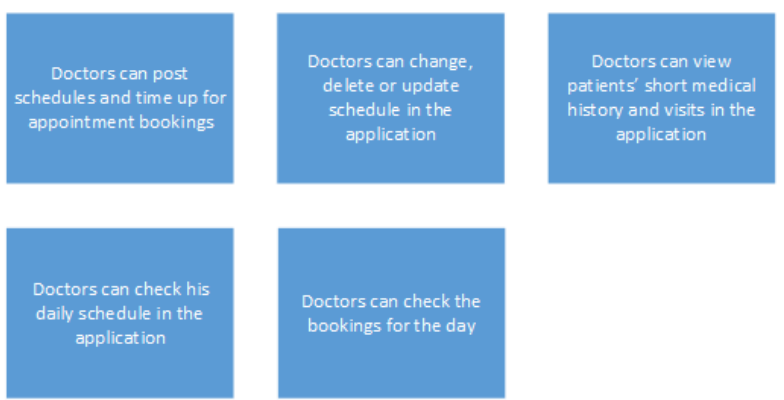

Fig. 5. Proposed functionalities for receptionist's side

With these proposed functionalities, this booking application will be able to support disaster management in various ways, especially in times when healthcare providers or specialised doctors can only be seen during certain times.

\subsection{Usability testing results}

The usability testing was conducted with 6 people via online video conference. More details on the users are shown in table 3.

Table 3 explains the demographic of the users. These users have agreed to be recorded and have asked to remain anonymous for this research.

Before the usability testing is carried out, a test plan must be devised. A test plan is a layout of how the usability testing will take place. The test plan will serve as a guide to the researcher to what activities and tasks must be carried out by the users. This test plan must be followed thoroughly. The goal is to discover any positive or negative findings within the application.

\subsubsection{Participants}

The participants of this usability testing are 6 people, where there were 3 patients user group, 1 doctor, 1 nurse/healthcare provider and 1 receptionist. Their demographics are shown in table 4.

Table 4. Participant demographics

\begin{tabular}{|l|l|}
\hline Item & Description \\
\hline Initials & RC \\
\hline Sex & Male \\
\hline Age & 21 \\
\hline User group & Patient \\
\hline & \\
\hline Initials & AU \\
\hline Sex & Female \\
\hline Age & 21 \\
\hline User group & Patient \\
\hline & \\
\hline Initials & SR \\
\hline Sex & Female \\
\hline Age & 21 \\
\hline User group & Patient \\
\hline & \\
\hline Initials & EW \\
\hline Sex & Female \\
\hline Age & 23 \\
\hline User group & Nurse/Healthcare provider \\
\hline & \\
\hline Initials & OR \\
\hline Sex & Male \\
\hline Age & 29 \\
\hline User group & Doctor/Healthcare provider \\
\hline & \\
\hline Initials & FM \\
\hline Sex & Female \\
\hline Age & 26 \\
\hline User Group & Receptionist \\
\hline & \\
\hline
\end{tabular}

All participants have agreed to be recorded during the usability testing process and they also agree that what they say during this process to be written or quoted within the research. 


\subsubsection{Working prototype}

Figure 6,7 and 8 are the working prototype implemented based on the vision of the application.

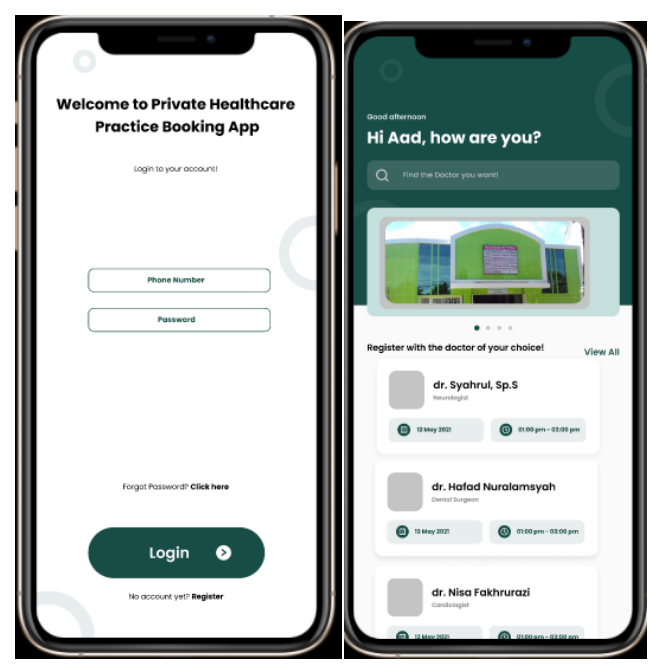

Fig. 6. Login and welcome page for patient's side of the application

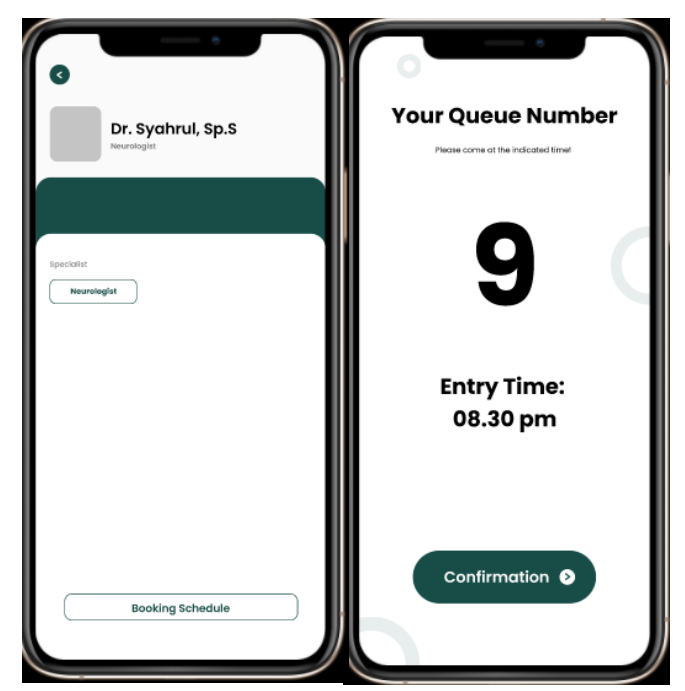

Fig. 7. Booking an appointment and getting the time slot for the appointment for patient's side of the application

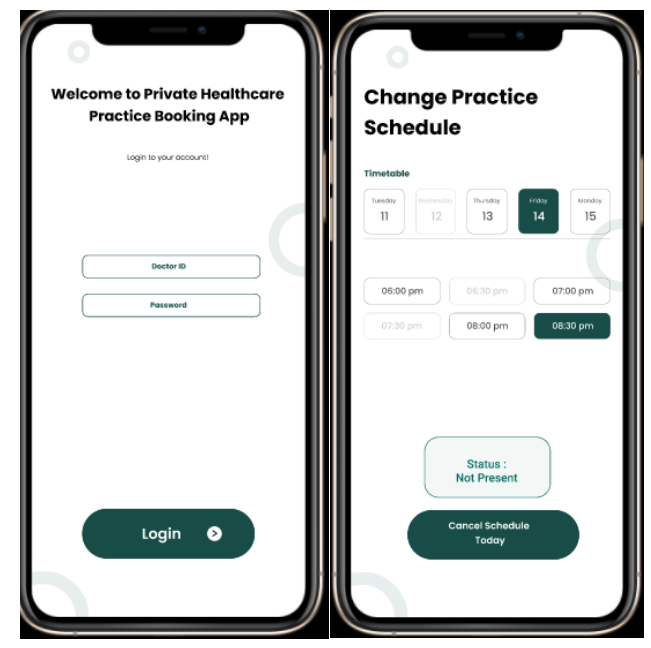

Fig. 8. Login and changing schedule for the doctor's side of the application

\subsubsection{Test plan}

The test plan for this application is laid out in table 5 .

Table 5. Test Plan for Booking Application

\begin{tabular}{|c|c|}
\hline Item & Description \\
\hline Q1 & $\begin{array}{l}\text { does the user understand how to use the } \\
\text { application without help from anyone? }\end{array}$ \\
\hline Goal & $\begin{array}{l}\text { user understands and able to use the } \\
\text { application without prior help }\end{array}$ \\
\hline Metric & $\begin{array}{l}\text { understand how to use the application } \\
\text { without prior help }\end{array}$ \\
\hline \multirow[t]{2}{*}{ Procedure } & $\begin{array}{l}\text { Ask users about their perception about the } \\
\text { application especially on the login, } \\
\text { registration and appointment number } \\
\text { functionalities }\end{array}$ \\
\hline & Ask users to conduct think aloud protocol \\
\hline $\begin{array}{l}\text { Data } \\
\text { collected }\end{array}$ & $\begin{array}{l}\text { Number of users able to understand and use } \\
\text { the application without help and comments } \\
\text { about the application }\end{array}$ \\
\hline Q2 & $\begin{array}{l}\text { does the user understand the goal of the } \\
\text { application? }\end{array}$ \\
\hline Goal & $\begin{array}{l}\text { User understands thoroughly on the goal and } \\
\text { objective of the application }\end{array}$ \\
\hline Metric & $\begin{array}{l}\text { Whether the user recognise the goal of the } \\
\text { application or not }\end{array}$ \\
\hline \multirow[t]{2}{*}{ Procedure } & $\begin{array}{l}\text { Ask users about the how-to in using the } \\
\text { application, for example using the } \\
\text { appointment function }\end{array}$ \\
\hline & Ask users to conduct think aloud protocol \\
\hline $\begin{array}{l}\text { Data } \\
\text { collected }\end{array}$ & $\begin{array}{l}\text { Observation on user's first impression about } \\
\text { the application and the number of users } \\
\text { recognise the goal of the application. }\end{array}$ \\
\hline Q3 & $\begin{array}{l}\text { Can the user group doctor/nurses/healthcare } \\
\text { provider clear his/her schedule without any } \\
\text { problem? }\end{array}$ \\
\hline Goal & $\begin{array}{l}\text { This user group can clear its schedule } \\
\text { without any problem }\end{array}$ \\
\hline Metric & This user group able to clear the schedule \\
\hline \multirow[t]{4}{*}{ Procedure } & Ask user to clear his/her schedule \\
\hline & Login to profile \\
\hline & Clear available schedule \\
\hline & Ask user to conduct think aloud protocol \\
\hline $\begin{array}{l}\text { Data } \\
\text { collected }\end{array}$ & $\begin{array}{l}\text { Time it takes to carry out the clear schedule } \\
\text { activity }\end{array}$ \\
\hline Q4 & $\begin{array}{l}\text { Can the user group administrator delete and } \\
\text { edit all data on patients and doctors without } \\
\text { any problem? }\end{array}$ \\
\hline Goal & $\begin{array}{l}\text { This user group can delete and edit data on } \\
\text { patients and doctors without any problem }\end{array}$ \\
\hline Metric & This user group is able to delete and edit data \\
\hline \multirow[t]{8}{*}{ Procedure } & Ask user to delete and edit data \\
\hline & Login to profile \\
\hline & Access patient's and doctor's data \\
\hline & Edit patient's data \\
\hline & Edit doctor's data \\
\hline & Delete patient's data \\
\hline & Delete doctor's data \\
\hline & Ask user to conduct think aloud protocol \\
\hline $\begin{array}{l}\text { Data } \\
\text { collected }\end{array}$ & $\begin{array}{l}\text { Time it takes to carry out the entire process } \\
\text { from start to finish }\end{array}$ \\
\hline
\end{tabular}


Table 4 explains a simplified version of the test plan for the booking application. The test was carried out thoroughly. The results as expressed in the next sections.

\subsubsection{Test plan results}

Q1 was asked to all participants. 4 out of 6 participants understood and able to use the application on the first glance. 2 participants did not understand how to use the application without help. The two participants who were not able to use the application; FM and SR on the first glance managed to use it again later after given some guidance.

Q2 was also carried out to all participants. 5 out of 6 participants understood the goal of the application. Only 1 participant did not get the main objective of the application. SR was the participant. During the application testing, SR was also the only one who had trouble in carrying out the booking functionality. She managed later on with some guidance.

Q3 was carried out to 2 of the participants in the doctor/nurse/healthcare provider user group, since the scheduling functionality is only available to this user group. Both participants, OR and EW were able to carry out the edit and delete process for scheduling without any hesitation. The time it took to conduct these functionalities are shown in table 6 .

Q4 was tested out 2 of the participants, FM and AU. Both are from different user groups. This is done in order to observe how users from different demographic can adapt with the administrative role. Both users were able to use edit, delete and conduct administrative functionalities with minimal intervention during the testing process. The time it took to conduct these functionalities are shown in table 7 .

\subsubsection{Think aloud findings}

During think aloud several negative and positive feedbacks were identified. From all 6 participants it was found that the simplicity of the application design made it easy to understand the functionalities provided by the application. Both receptionist and healthcare provider user group also agreed that the design of the application made them easier to recognise the goal of the application, without any previous experience.

The negative point of this application that was identified during the think aloud protocol was that the navigation of the application was not clear. This was emphasised the most in the patient user group. Even when 4 participants from this user group operated the application with general ease, there were repeated comments about the navigation of the application. One participant said "I am not sure where to begin booking for a doctor after the login page" (SR, 21).

This suggests that the user experience needs to be redesign in the future with the consideration of a wider demographic area. This result suggests that SR may need a different approach in user experience.

During the entire procedure, every participant agrees that this application will be helpful during a disaster and a pandemic. Even though both situations are dire, people with certain diseases are still present. The people are still needed to be treated. OR, 29 is a doctor. He thought that this application is extremely important to be installed in private doctors' practices. In his practice, he has many patients who are waiting in line to meet the doctors during appointment. During the pandemic, the regulation is that there should be minimal crowd and there should be a distance about 1.5 to 2 metres between people. This application can also help uphold this regulation and reduce the number of people in the private practices.

This application is also resilient. This was mentioned by FM. She had two perspectives for this application. One as a patient. During a disaster, as it happened previously in Banda Aceh. It was a lot harder to book an appointment to meet specialised doctors. Based on her own experience as a receptionist, this application is useful during emergency situation, such as during a disaster, as it can help patients book a time that is convenient for them to meet a doctor.

\section{Conclusion}

To conclude the design and testing of this booking application has been prolific. Necessary data has been gathered and these data can also be a starting point for future development of this application. Using UCD as the design methodology has proven to be effective, as proven in the usability testing, where all 6 participants have agreed that simple technology such as this booking application can help them in the day-to-day tasks in the specialised doctor's practice.

\section{References}

1. G. Shank, Qualitative Research: A Personal Skills Approach, 2nd ed. (Pearson, USA, 2005)

2. S. D. Lapan and F. J. Riener, Qualitative Research an Introduction to Methods and Designs, 1 st ed. (Jossey-Bass, San Francisco, 2012)

3. B. Martin and B. Hanington, Universal Methods of Design, 2012th ed. (Rockport Publishers, Berverly, Massachusets, n.d.)

4. N. K. Denzin, K. Norman, and Y. S. Lincoln, Handbook of Qualitative Research, 1st ed. (Sage Publications, California, 2009)

5. L. Mckechnie, L. Baker, M. Greenwood, and H. Julien, New Review of Information Behaviour Research 3, 113 (2002)

6. L. H. Rosen and M. K. Underwood, in Encyclopedia of Research Design, edited by N. Salkind J., 1st ed. (Sage Publications, California, 2010), pp. 952-954

7. L. Brinkmann, in Interviewing: Understanding Qualitative Research, 1st ed. (Oxford University Press, New York, 2013), pp. 26-32

8. L. Benaquisto, in The SAGE Encyclopedia of Qualitative Research Methods, edited by L. Given M. (Sage Publications, USA, 2008), pp. 85-88

9. M. Endsley R., B. Bolte, and D. Jones G., Designing for Situation Awareness: An Approach 
to User-Centered Design (Taylor \& Francis, Georgia, 2003)

10. M. Tara, S. Taneva, M. Casselman, M. Yeung, C. McDaniel, W. Ho, and J. Cafazzo, BIT 46, 49 (2021)

11. K. Holtzblatt, J. B. Wendell, and S. Wood, Rapid Contextual Design: A How-To Guide to Key Techniques for User-Centred Design, 1st ed. (Elsevier, USA, 2005)

12. H. Sharp, Y. Rogers, and Jennifer Preece, Interaction Design: Beyond Human-Computer Interaction, 5th ed. (John Wiley \& Sons, Indianapolis, 2019) 